\title{
Musical murmur in a dog with acute chordae tendineae rupture
}

\author{
Sa-Hee Min, Min-Hee Kang, Hee-Myung Park* \\ Department of Veterinary Internal Medicine, College of Veterinary Medicine, Konkuk University, Seoul 143-701, Korea
}

(Received: April 4, 2013; Accepted: August 26, 2013)

\begin{abstract}
A 6 year-old, spayed female, Maltese dog was presented with precordial thrill and mild coughing. Thoracic auscultation revealed a grade V/VI systolic murmur with maximal intensity over the left apex characterized by musical murmur. Echocardiography revealed mild myxomatous degeneration of mitral valve and ruptured chordae tendineae. Musical murmur was produced due to the vibration of ruptured piece of chordae tendineae along with regurgitant flow. After treatment with furosemide and ramipril, clinical signs resolved and precordial thrill reduced. This case report describes typical clinical signs and phonocardiogram of musical murmur in a dog with acute chordae tendineae rupture.
\end{abstract}

Keywords : chordae tendineae rupture, mitral valve disease, musical murmur

Mitral valve disease (MVD) is the most common acquired heart disease in small breed-dogs and characterized by myxomatous valvular degeneration $[3,5,6,7,8]$. It results in mitral valve insufficiency creating systolic mitral regurgitation, which in turn leads to a left apical systolic murmur [7]. Usually with MVD, systolic murmur intensity is related to disease severity. However, occasionally with mild stage of MVD, the murmur has a musical quality and striking intensity [9].

This case describes sudden onset of precordial thrill characterized as musical murmur resulted from acutely ruptured chordae tendineae with midly degenerated mitral valve.

A 6 year-old, spayed female, Maltese dog was presented with sudden onset of precordial thrill and mild nocturnal coughing. Thoracic auscultation revealed a grade V/VI systolic murmur with maximal intensity over the left apex. Laboratory data including a complete blood count, serum biochemical analysis were within normal ranges. Evaluation of the electrocardiogram showed mildly prolonged $\mathrm{P}$ wave. Thoracic radiography revealed bronchointerstitial pattern in bilateral caudal lung lobes. 2D-echocardiography demonstrated slightly thickened mitral valve and pieces of ruptured chordae tendineae attached to the tip of mitral valve leaflet (Fig. 1). Doppler echocardiography revealed mild systolic turbulent flow in the left atrium running around atrial wall with peak velocity $2.07 \mathrm{~m} / \mathrm{sec}$ and pressure gradient 17.2 $\mathrm{mmHg}$ (Fig. 1). There was no marked evidence of cardiac remodeling. Phonocardiography was performed, and showed mid-systolic diamond shape murmur with variable amplitudes (Fig. 2). Recorded sound files of phonocardiogram revealed sharp, pitched 'wheeping' sound, characterized as musical murmur. The blood sample collected aseptically was submitted for aerobic and anaerobic blood culture, and the result was negative. Based on the physical and clinical examination, the dog was diagnosed as mitral valve degeneration (MVD) with chordae tendineae rupture (CTR). Furosemide (Lasix, $0.5 \mathrm{mg} / \mathrm{kg}$, oral, twice daily; Handok phrmaceuticals, Korea) and ramipril (Vasotop, $0.125 \mathrm{mg} / \mathrm{kg}$, oral, once a daily; Intervet, Korea) were administered. After 1 month of medical treatment, nocturnal coughing was improved and precordial thrill was reduced.

Generally, musical murmur is characterized by a striking musical quality and is frequently so loud that it can be heard over the entire thorax. The murmur has been described in human literature using following terms: "cooing of a dove,", "buzzing of a saw", "like a cuckoo clock," "like the spinning of a top" [1]. Musical murmur would occur owing to the resonance of vibrating structure projecting into the regurgitant blood flow. In human medicine, musical murmur is most frequently occurred in aortic stenosis [4]. In the present case, it was ruptured piece of chordae tendineae flailed with mitral valve during systole vibrating with turbulent blood flow that made loud, pinched sound. The size of turbulent jet flow was relatively small and ran around the atrial wall. The direction of jet flow indicated that the degeneration of anterior leaflet was worse than posterior leaflet.

Historically, CTR is commonly described as a devastating complication of MVD, because it is responsible for acute left heart failure leading to pulmonary edema and death $[2,8]$. However, one study suggested that $24.6 \%$ of the dogs with CTR were asymptomatic and therefore assigned to International Small Animal Health Council (ISACHC) classes Ia and $\mathrm{Ib}$ at the time of diagnosis [8]. In this case, 2-D echocar-

*Corresponding author

Tel: +82-2-450-4140, Fax: +82-2-444-4396

E-mail: parkhee@konkuk.ac.kr 

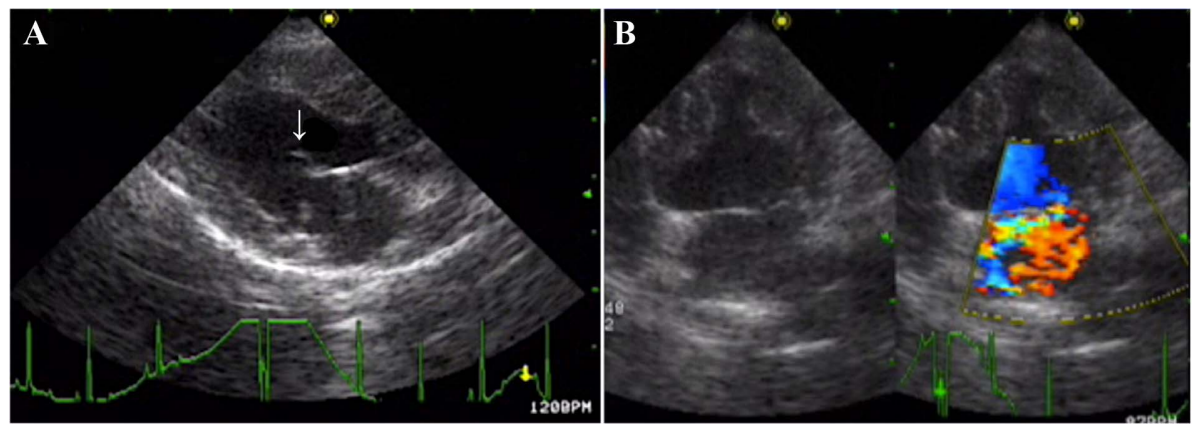

Fig. 1. Echocardiogram of the paient. (A) 2-D echocardiogram (right parasternal 5-chamber view), ruptured piece of chordae tendineae (arrow) flails with degenerated mitral valve. (B) Color Doppler echocardiogram (left caudal parasternal 4-chamber view) showed mitral regurgitant turbulent jet run around left atrial wall.

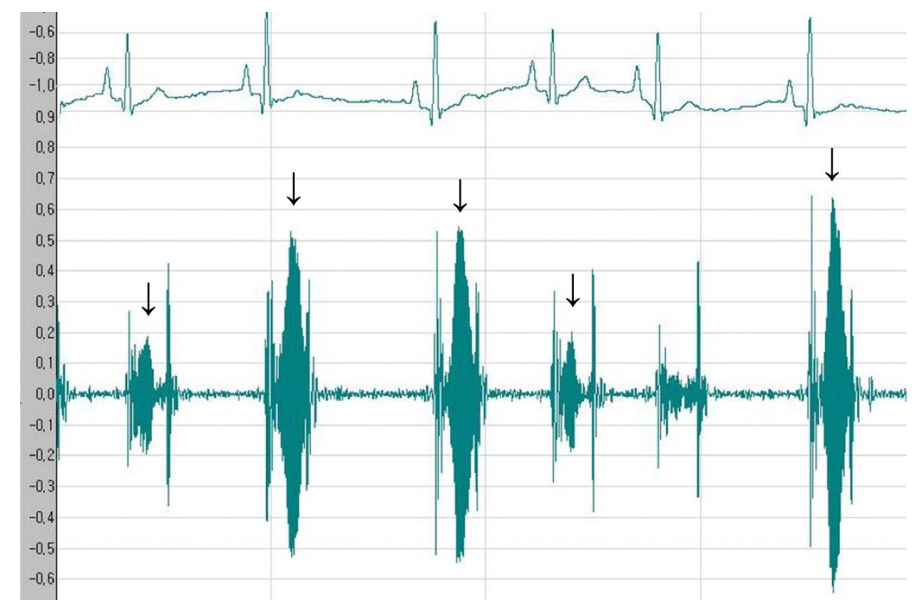

Fig 2. Phonocardiography of systolic, diamond shape and musical quality murmur (arrows) recorded at the mitral area.

diography showed mitral valve degeneration and linear structures that attached to the tip of anterior leaflet suggesting CTR. This dog had mild clinical sign and showed no evidence of cardiac remodeling, and thus assigned to early course of ISACHC II. Generally, medical intervention is indicated when overt clinical signs of heart failure showed (ISACHC $\geq$ II) [3]. The dog excellently responded to therapy and intensity of murmur reduced due to decreased size of turbulent jet flow.

In conclusion, this report provides evidence of acute chordae tendineae rupture, even with mild degeneration of mitral valve. Acutely ruptured chordae tendineae could produce sudden onset of strikingly loud, high-pitched murmur regardless of clinical sign severity.

\section{References}

1. Gelfand D, Bellet $\mathbf{S}$. The musical murmur of aortic insufficiency: clinical manifestations; based on a study of 18 cases. Am J Med Sci 1951, 221, 644-654.

2. Kittleson MD. Myxomatous atrioventricular valvular degeneration. In: Kittleson MD, Kienle RD (eds.). Small Animal Cardiovascular Medicine. pp. 297-318, Mosby, St. Louis, 1998.

3. Lombard CW, Jöns O, Bussadori CM. Clinical efficacy of pimobendan versus benazepril for the treatment of acquired atrioventricular valvular disease in dogs. $\mathrm{J}$ Am Anim Hosp Assoc 2006, 42, 249-261.

4. Miyahara K, Amitani S, Sohara H, Kurose M, Iwamura H, Toyohira H, Taira A. Chaotic musical murmur in aortic regurgitation. Jpn Circ J 1996, 60, 993-997.

5. Olsen LH, Häggström J, Peterson HD. Acquired valvular heart disease. In: Ettinger SJ, Feldman EC (eds.). Textbook of Veterinary Internal Medicine: Disease of the Dog and Cat. 7th ed. vol 2. pp. 1299-1319, Saunders, St. Louis, 2010.

6. Pedersen HD, Häggström J. Mitral valve prolapse in the dog: a model of mitral valve prolapse in man. Cardiovasc Res 2000, 47, 234-243.

7. Serfass P, Chetboul V, Sampedrano CC, Nicolle A, Benalloul T, Laforge H, Gau C, Hébert C, Pouchelon JL, Tissier R. Retrospective study of 942 small-sized dogs: prevalence of left apical systolic heart murmur and leftsided heart failure, critical effects of breed and sex. $\mathrm{J}$ Vet Cardiol 2006, 8, 11-18.

8. Serres F, Chetboul V, Tissier R, Sampedrano CC, Gouni V, Nicolle AP, Pouchelon JL. Chordae tendineae rupture in dogs with degenerative mitral valve disease: prevalence, survival, and prognostic factors (114 cases, 2001-2006). J Vet Intern Med 2007, 21, 258-264.

9. Ware WA. Clinical manifestations of cardiac disease. In: Nelson RW, Couto CG (eds.). Small Animal Internal Medicine. 4th ed. pp. 1-11, Mosby, St. Louis, 2009. 